\title{
Mathematical Modeling and Inverse Analysis for Microbial Depolymerization Processes of Xenobiotic Polymers
}

\author{
Masaji Watanabe ${ }^{*}$, Fusako Kawai2 \\ ${ }^{1}$ Faculty of Mathematics and Natural Sciences, Andalas University, Padang, Republic of Indonesia \\ ${ }^{2}$ Okayama University, Okayama, Japan \\ Email: ${ }^{\star}$ watan-m@okayama-u.ac.jp
}

How to cite this paper: Watanabe, M. and Kawai, F. (2019) Mathematical Modeling and Inverse Analysis for Microbial Depolymerization Processes of Xenobiotic Polymers. Journal of Materials Science and Chemical Engineering, 7, 39-46.

https://doi.org/10.4236/msce.2019.712005

Received: October 14, 2019

Accepted: December 17, 2019

Published: December 20, 2019

\begin{abstract}
Microbial depolymerization processes of xenobiotic polymers are discussed. A mathematical model is formulated and inverse problems for a time factor and a molecular factor of a degradation rate are described. Experimental outcomes are introduced in inverse analyses. Once the time factor and the molecular factor are obtained, the microbial depolymerization process is simulated. Numerical techniques are illustrated and numerical results are presented.
\end{abstract}

\section{Keywords}

Microbial Depolymerization, Polyetylene Glycol 4000, Mathematical Model, Inverse Problem, Numerical Simulation

\section{Introduction}

Polyethylene (PE) and polyethylene glycol (PEG) are exogenously depolymerizable. In exogenous type depolymerization processes, molecules liberate small units from their terminals. Microbial depolymerization processes of PEG have been documented in detail. Studies of PEG biodegradation include utilization of PEG of average molecular weight 20,000 by Psedonomas aeruginosa [1], degradation of PEG 20,000 by anaerobic bacteria isolated from sludge of a municipal anaerobic digester [2], and confirmation of efficient biodegradation of PEG by Pseudomonas stutzeri [3]. Microbial depolymerization processes of PE and PEG have also been studied mathematically. A mathematical model was proposed in numerical studies of biodegradation of PE [4]. The mathematical techniques devised for PE biodegradation processes were applied to biodegradation processes of 
PEG [5].

Molecules are broken down randomly in an endogenous type depolymerization process. Polyvinyl alcohol (PVA) and polylactic acid (PLA) are depolymerizable in endogenous type depolymerization processes. A mathematical model was proposed for enzymatic degradation process of PVA [6]. The mathematical techniques devised for PVA were applied to an enzymatic hydrolysis of polylactic acid (PLA) [7]. Techniques originally devised for endogenous type microbial depolymerization processes were applied to exogenous type depolymerization processes of PE and PEG [8].

In this study, mathematical techniques devised in previous studies were reapplied to a depolymerization process of PEG 4000. Weight distribution of PEG 4000 before and after cultivation of Sphingopyxis macrogoltabidus strain 103 were applied in inverse analyses for a molecular factor and a time factor of a degradation rate.

\section{Description of Mathematical Formulation for Exogenous Type Depolymerization Process}

Let $w(t, M)[\mathrm{mg}]$ be the weight distribution of a polymer with respect to the molecular weight $M$ at time $t$, and let $v(t)[\mathrm{mg}]$ be the total weight of polymer molecules with molecular weight between $A$ and $B$ at time $t$. The total weight $v(t)$ over the interval $[A, B]$ is expressed in terms of the integral of $w(t, M)$ from $A$ to $B$,

$$
v(t)=\int_{A}^{B} w(t, M) d M
$$

In particular, the total weight $v(t)$ of the entire residual polymer at time $t$ is expressed in terms of the integral of $w(t, M)$ over the interval $[0, \infty)$

$$
v(t)=\int_{0}^{\infty} w(t, M) d M
$$

Integral (1) is a reasonable approximation of the integral (2) with suitable values of $A$ and $B$. In this study, integral (2) over the infinite interval (2) was replaced by an integral over a finite interval (1) with $A=10^{3.5}$ and $B=10^{3.75}$. Similarly, an integral with the lower limit 0 was replaced by an integral with the lower limit $A=10^{3.5}$, and an integral with the upper limit $\infty$ was replaced by an integral with the upper limit $B=10^{3.75}$.

Suppose that $\sigma(t)$ is the population of viable cells at time $t$ in a medium in which the polymer is the sole carbon source. The system of Equations (3), (4) for the weight distribution $w(t, M)$ and the microbial population $\sigma(t)$ was proposed in previous studies [9]-[14].

$$
\begin{gathered}
\frac{\partial w}{\partial t}=\sigma(t)\left[-\lambda(M) w+c(M) \int_{M}^{\infty} \lambda(K) d(K) w(t, K) d K\right] \\
\frac{d \sigma}{d t}=k\left[-v^{\prime}(t)\right]-h \sigma, \\
c(M)=M e^{\rho M}, d(K)=\frac{\rho e^{-\rho K}}{K\left(1-e^{-\rho K}\right)}, \rho=\frac{\log 2}{L} .
\end{gathered}
$$


The parameter $L$ is the molecular weight of a monomer unit, e.g. PE: $L=28$ $\left(\mathrm{CH}_{2} \mathrm{CH}_{2}\right)$, PEG: $L=44\left(\mathrm{CH}_{2} \mathrm{CH}_{2} \mathrm{O}\right)$, and $k$ and $h$ are positive parameters. The function $\lambda(M)$ is the molecular factor of degradation rate, and the function $\sigma(t)$ is the time factor of degradation rate. Equations (2) and (3) lead to the expression for the derivative of $v(t)$,

$$
v^{\prime}(t)=\sigma(t) \int_{0}^{\infty}\left[-\lambda(M) w(t, M)+c(M) \int_{M}^{\infty} \lambda(K) d(K) w(t, K) d K\right] d M
$$

System of Equations (3), (4) for $w(t, M)$ and $\sigma(t)$ is associated with the initial conditions

$$
\begin{gathered}
w(0, M)=f_{0}(M) \\
\sigma(0)=\sigma_{0}
\end{gathered}
$$

where $f_{0}(M)$ and $\sigma_{0}$ are the initial weight distribution and the initial microbial population, respectively.

\section{Inverse Problems for Molecular Factor and Time Factor of Degradation Rate and Their Solutions}

Once the molecular factor $\lambda(M)$ and values of parameters $\sigma_{0}, k$, and $h$ are set, initial value problem (3), (4), (6), (7) becomes solvable a problem for unknown functions $w(t, M)$ and $\sigma(t)$. In order to obtain the function $\lambda(M)$ and values of parameters $\sigma_{0}, k$, and $h$, the change of variables from $t$ to $\tau$ (8) was applied.

$$
\tau=\int_{0}^{t} \sigma(s) d s
$$

Suppose that $W(\tau, M), S(\tau)$, and $V(\tau)$ are functions which correspond to $w(t, M), \sigma(t)$, and $v(t)$, respectively, according to the change of variables (8), that is

$$
W(\tau, M)=w(t, M), \quad S(\tau)=\sigma(t), V(\tau)=v(t) .
$$

In view of the expression

$$
\frac{\partial}{\partial \tau}=\frac{\partial}{\partial t} \frac{\partial t}{\partial \tau}=\frac{1}{\sigma(t)} \frac{\partial}{\partial \tau}
$$

equations (3) and (4) lead to

$$
\begin{gathered}
\frac{\partial W}{\partial \tau}=-\lambda(M) W+c(M) \int_{M}^{\infty} \lambda(K) d(K) W(\tau, K) d K, \\
\frac{d S}{d \tau}=-k V^{\prime}(\tau)-h,
\end{gathered}
$$

respectively. The expression

$$
v^{\prime}(t)=\frac{d v}{d t}=\frac{d V}{d \tau} \frac{d \tau}{d t}=V^{\prime}(\tau) \sigma(t),
$$

leads to the equation

$$
V^{\prime}(\tau)=\frac{v^{\prime}(t)}{\sigma(t)}
$$


Expressions (5) and (10) lead to

$$
V^{\prime}(\tau)=\int_{0}^{\infty}\left[-\lambda(M) W(\tau, M)+c(M) \int_{M}^{\infty} \lambda(K) d(K) W(\tau, K) d K\right] d M .
$$

Let $F_{1}(M)$ be the weight distribution for $\tau=T_{1}$ so that

$$
W\left(T_{1}, M\right)=F_{1}(M)
$$

and $F_{2}(M)$ be the weight distribution for $\tau=T_{2}$ so that

$$
W\left(T_{2}, M\right)=F_{2}(M) .
$$

Equation (9), the initial condition (12), and the terminal condition (13) form an inverse problem for $\lambda(M)$, for which the solution of the initial value problem (9), (12) also satisfies the terminal condition (13). Numerical techniques for the inverse problem (9), (12), (13) were devised in previous studies. Those techniques were applied to weight distributions of PEG 4000 before and after cultivation of Sphingopyxis macrogoltabidus strain 103 for one day, three days, five days, and seven days [15]. Figure 1 shows the molecular factor $\lambda(M)$ of degradation rate based on weight distributions before after cultivation of the microorganism for three days.

Once the inverse problem for $\lambda(M)$ was solved, Equation (9) was solved for $W(\tau, M)$ with the initial condition $W\left(\tau_{0}, M\right)=f_{0}(M)$, where $\tau_{0}=0$. Figure 2 shows the curve $(\tau, V(\tau) / V(0) \times 100)$. The numerical results show that the value of $t, t_{0}=0, t_{1}=1, t_{2}=3, t_{3}=5$, and $t_{4}=7$ correspond to the value of $\tau, \tau_{0}=0, \tau_{1} \approx 0.009, \tau_{2} \approx 1.187, \tau_{3} \approx 5.569$, and $\tau_{4} \approx 8.448$.

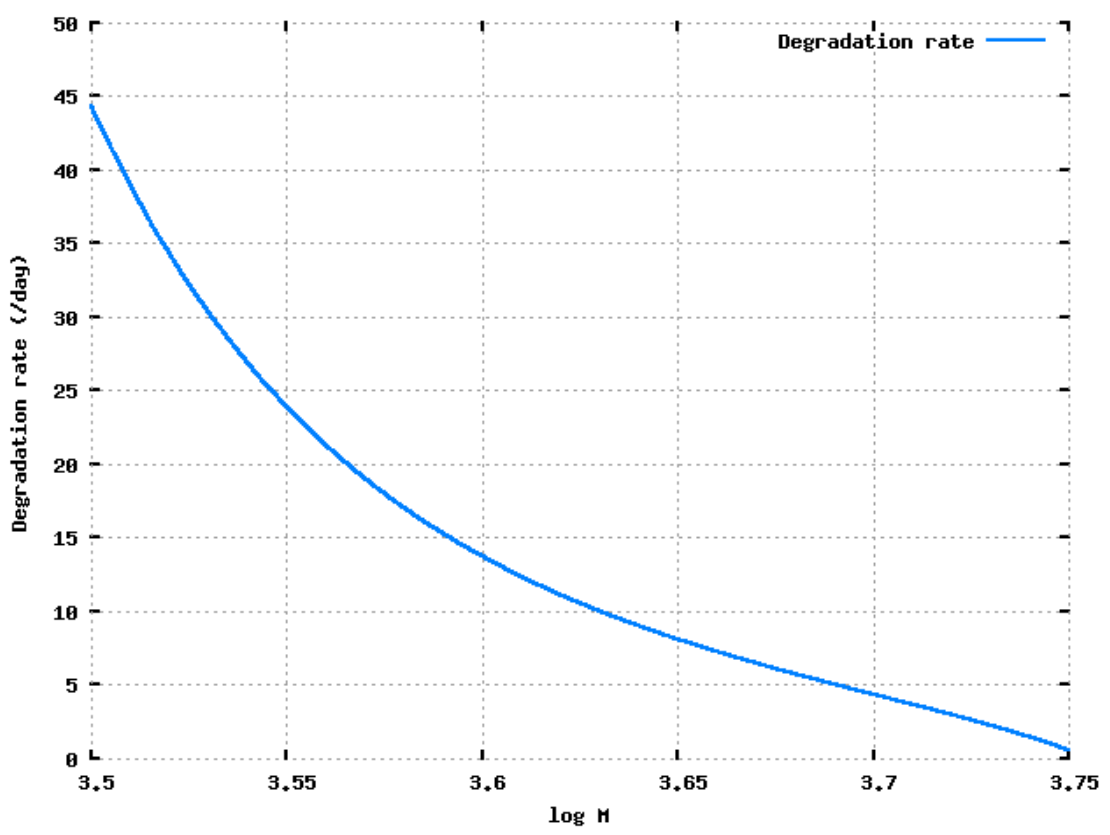

Figure 1. This figure shows the molecular factor of degradation rate based on weight distributions before and after cultivation of Sphingopyxis macrogoltabidus strain 103 for three days. 


\section{Application of Numerical Techniques to Inverse Problem for Time Factor of Degradation Rate}

According to a previous study, function $V(\tau)$ is well approximated by an exponential function

$$
V(\tau)=v_{0} e^{-\mu \tau} \quad\left(v_{0}=\int_{0}^{\infty} f_{0}(M) d M\right),
$$

so that $V^{\prime}(\tau)=-\mu v_{0} e^{-\mu \tau}$. In this study, function $V(\tau)$ was replaced with the exponential function (14) with a value of the parameter $\mu$ approximately equal to 0.574436 , and equations $V\left(\tau_{i}\right)=v\left(t_{i}\right)(i=1,2,3,4)$ were solved numerically.

Let $S\left(\tau, \sigma_{0}, k, h\right)$ be the solution of the equation (10) with initial value $\sigma_{0}$. The change of variables (8) leads to the expression $t=q\left(\tau, \sigma_{0}, k, h\right)$, where

$$
q\left(\tau, \sigma_{0}, k, h\right)=\int_{0}^{\tau} \frac{d r}{S\left(r, \sigma_{0}, k, h\right)} .
$$

Given $m$ pairs of values of $t$ and $\tau,\left(t_{i}, \tau_{i}\right)(i=1, \ldots, m)$, equations for $\sigma_{0}, k$, and $h$,

$$
g_{i}\left(\sigma_{0}, k, h\right)=0 \quad(i=1, \cdots, m)
$$

where $g_{i}\left(\sigma_{0}, k, h\right)=q\left(\tau_{i}, \sigma_{0}, k, h\right)-t_{i}$, were set. Each of intervals $I_{1}=[0.001,0.1]$, $I_{2}=[0.003,0.02]$, and $I_{3}=[0.001,0.2]$, for $\sigma_{0}, k$, and $h$, respectively, was divided into one hundred equally spaced subintervals, and the error

$$
\sqrt{\left\{g_{2}\left(\sigma_{0}, k, h\right)\right\}^{2}+\left\{g_{3}\left(\sigma_{0}, k, h\right)\right\}^{2}}
$$

was evaluated at the $101^{3}$ combinations of the nodal points, among which

$$
\left(\sigma_{0}, k, h\right) \approx(0.01684,0.0064,0.11642)
$$

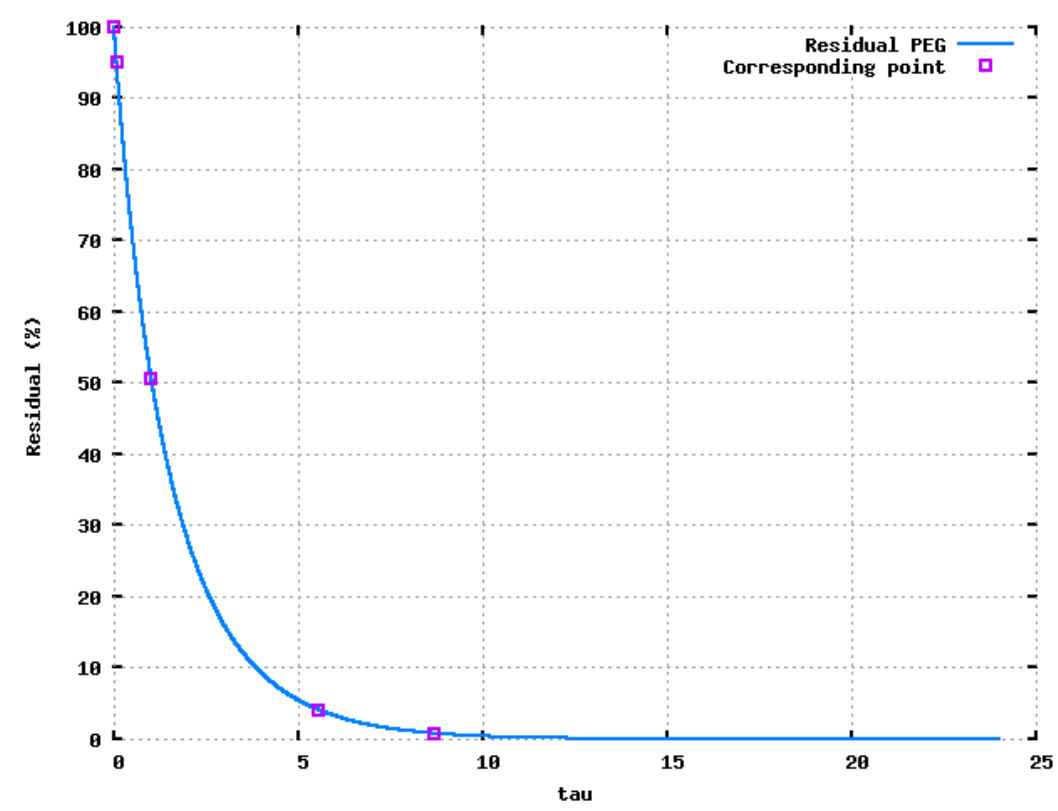

Figure 2. CURVE $(\tau, V(\tau) / V(0) \times 100)$. 
gave the minimum value to the error. Figure 3 shows the curve $(t, \sigma(t))=\left(q\left(\tau, \sigma_{0}, k, h\right), S\left(\tau, \sigma_{0}, k, h\right)\right)$ for those values of $\sigma_{0}, k$, and $h$. In the previous study, initial value problem (3), (6) was solved numerically, once the time factor $\sigma(t)$ was obtained. In this study, initial value problem (3), (4), (6), (7) was solved numerically. Figure 4 shows experimental and numerical results for the transition of the total weight distributions of PEG 4000 during the cultivation of Sphingopyxis macrogoltabidus strain 103 for seven days.

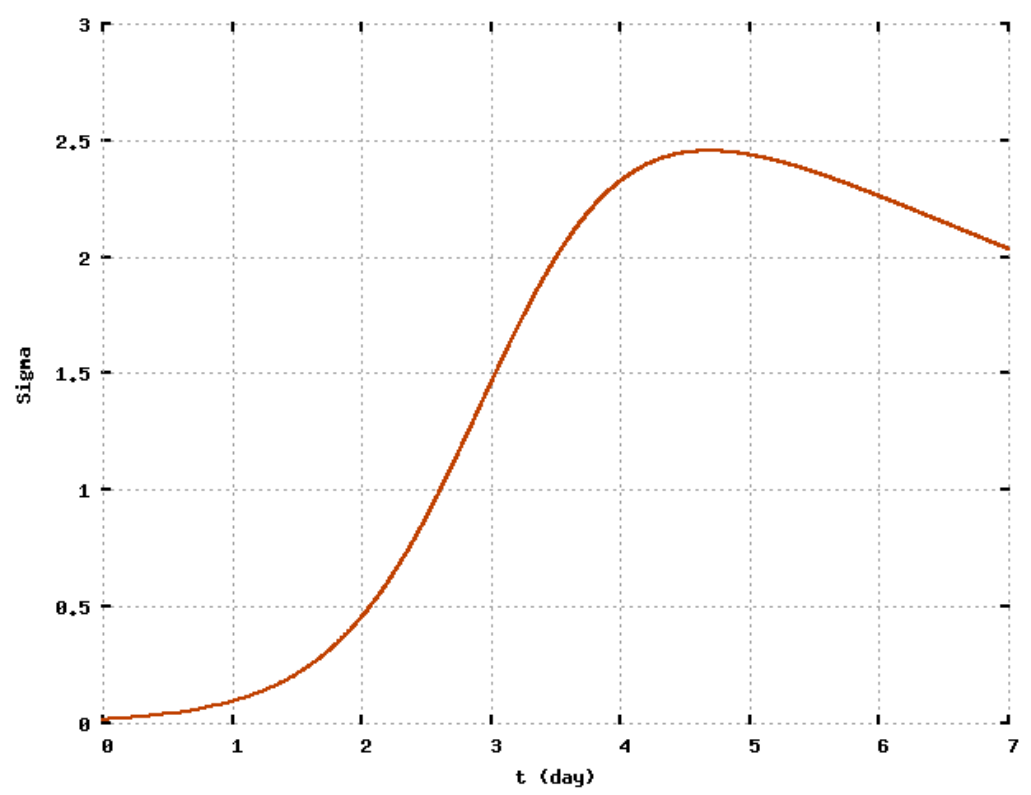

Figure 3. Curve $(t, \sigma(t))=\left(q\left(\tau, \sigma_{0}, k, h\right), S\left(\tau, \sigma_{0}, k, h\right)\right)$ for $\left(\sigma_{0}, k, h\right) \approx(0.01684,0.0064,0.11642)$.

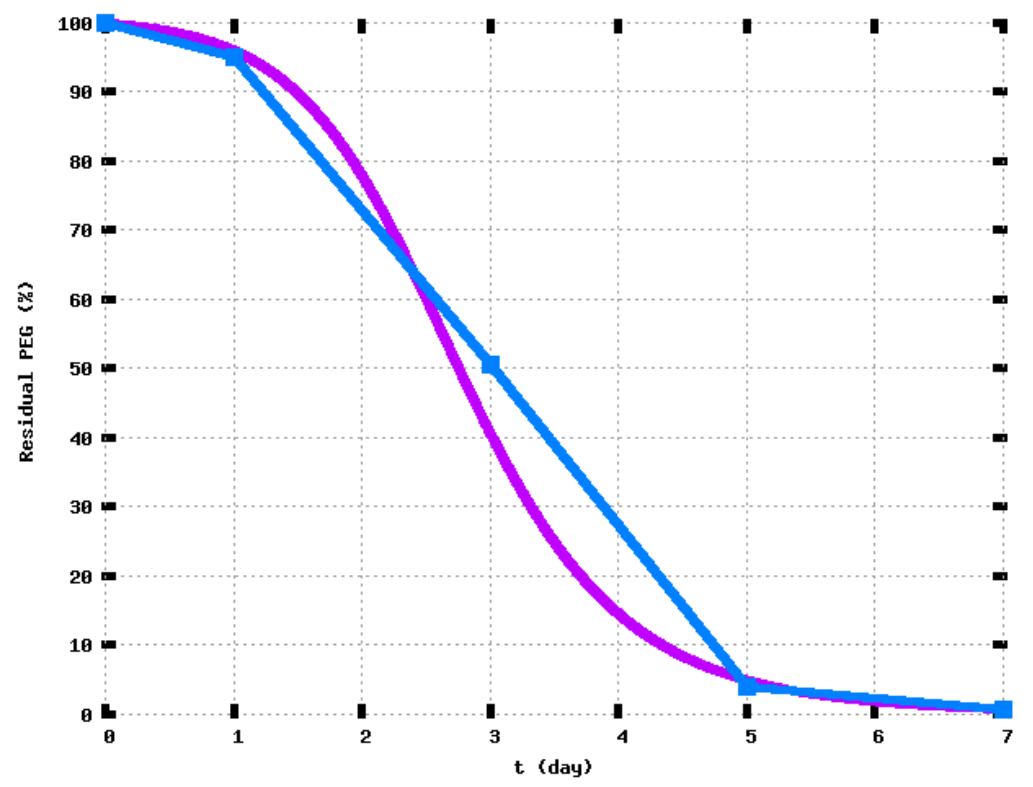

Figure 4. Transition of the total weight of PEG 4000 during cultivation of Sphingopyxis macrogoltabidus strain 103 for seven days. 


\section{Discussion}

Mathematical techniques for depolymerization processes were demonstrated. In particular, exogenous type depolymerization process of PEG 4000 was analyzed. The weight distributions of PEG 4000 before and after cultivation of Sphingopyxis macrogoltabidus strain 103 for one day, three days, five days, and seven days are introduced into the analysis. Figure 1 shows the molecular factor based on the weight distributions before and after cultivation for three days. The inverse problem for the time factor of the degradation rate was solved (Figure 3). Once the molecular factor and values of the parameters that determine the time factor of the degradation rate were obtained, the initial value problem (3), (4), (6), (7) was solved numerically, and the exogenous type depolymerization process of PEG 4000 was simulated.

\section{Acknowledgements}

The authors thank Ms. Y. Shimizu and Dr. S. Jimbo for their technical support.

\section{Conflicts of Interest}

The authors declare no conflicts of interest regarding the publication of this paper.

\section{References}

[1] Haines, J.R. amd Alexander, M. (1975) Microbial Degradation of Polyethylene Glycol. Applied Microbiology, 621-625.

[2] Dwyer, D.F. and Tiedje, J.M. (1983) Degradaton of Ethylene Glycol and Polyethylene Glycols by Methanogenic Consortia. Applied and Environmental Microbiology, 185-190.

[3] Obradors, N. and Aguilar, J. (1991) Efficient Biodgradation of High-Molecular-Weight Polyethylene Glycols by Pure Cultures of Pseudomonas Stutzeri. Applied and Environmental Microbiology, 2383-2388.

[4] Watanabe, M., Kawai, F., Shibata, M., Yokoyama, S., Sudate, Y. and Hayashi, S. (2004) Analytical and Computational Techniques for Exogenous Depolymerization of Xenobiotic Polymers. Mathematical Biosciences, 192, 19-37. https://doi.org/10.1016/j.mbs.2004.06.006

[5] Watanabe, M. and Kawai, F. (2009) Effects of Microbial Population in Degradation Process of Xenobiotic Polymers. In: Howlett, P., Nelson, M. and Roberts, A.J., Eds., Proceedings of the 9 th Biennial Engineering Mathematics and Applications Conference, EMAC-2009, Vol. 51 ANZIAM J, C682-C96. http://journal.austms.org.au/ojs/index.php/ANZIAMJ/article/view/2433 https://doi.org/10.21914/anziamj.v51i0.2433

[6] Watanabe, M. and Kawai, F. (2006) Mathematical Modelling and Computational Analysis of Enzymatic Degradation of Xenobiotic Polymers. Applied Mathematical Modelling, 30, 1497-1514. https://doi.org/10.1016/j.apm.2005.12.011

[7] Watanabe, M., Kawai, F., Tsuboi, S., Nakatsu, S. and Ohara, H. (2007) Study on Enzymatic Hydrolysis of Polylactic Acid by Endogenous Depolymerization Model. Macromolecular Theory and Simulations, 16, 619-626. 
https://doi.org/10.1002/mats.200700015

[8] Watanabe, M. and Kawai, F. (2014) Simulation for Microbial Depolymerization Processes of Polyethylene Glycol. In: Gaol, F.L., Soewito, B., Bououdina, P., Chen, M.-S., Eds., Proceedings of the 2013 International Conference on Advances in Intelligent Systems in Bioinformatics, intel-13, Advances in Intelligent Systems Research, Atlantis Press, February 2014, 1951-6851.

https://www.atlantis-press.com/proceedings/intel-13/11361 https://doi.org/10.2495/intelsys130111

[9] Watanabe, M. and Kawai, F. (2016) Simulation of Microbial Depolymerization Process with Exponential Consumption of Carbon Source. 2016 International Conference on Computer, Mechatronics and Electronic Engineering (CMEE 2016), DEStech Publications, Inc., Lancaster, Pennsylvania, 426-431. https://doi.org/10.12783/dtcse/cmee2016/5356

[10] Watanabe, M. and Kawai, F. (2017) Computational Analysis for Biodegradation of Exogenously Depolymerizable Polymer. 2017 The 5 th International Conference on Mechanical Engineering, Material Science and Civil Engineering, December 2017, Kuala Lumpur, Malaysia, 012015, IOP Conference Series: Materials Science and Engineering, Published Online: 6 April 2018. IOP Publishing. https://doi.org/10.1088/1757-899x/324/1/012015

[11] Watanabe, M. and Kawai, F. (2018) Computational Study of Bacterial Depolymerization Process of Xenobiotic Polymers. 2018 2nd International Conference on Material Engineering and Advanced Manufacturing Technology (MEAMT 2018), Article Number 02007, p. 7, Section Material Simulation. https://doi.org/10.1051/matecconf/201818902007

[12] Watanabe, M. and Kawai, F. (2018) Numerical Simulation of Exogenous Type Microbial Depolymerization Process with Weight Distributions and Microbial Population. Proc. of The Seventh Intl. Conf. on Advances in Civil, Structural and Environmental Engineering-ACSEE 2018, 19-22.

[13] Watanabe, M. and Kawai, F. (2018) Exponential Approximation of Consumption Rate of Carbon Source in Numerical Simulation of Microbial Depolymerization Process. International Journal of Advances in Science Engineering and Technology, 7.

[14] Watanabe, M. and Kawai, F. (2019) Mathematical Modeling and Inverse Analysis for Microbial Depolymerization Processes of Xenobiotic Polymers. Journal of Materials Science and Chemical Engineering, 7, 39-46.

[15] Watanabe, M. and Kawai, F. (2018) Inverse Analysis for Microbial Depolymerization Process of Polyethylen Glycol 4000. 2018 International Conference on Computer, Communications and Mechanical Engineering (CCME 2018). https://doi.org/10.12783/dtcse/ccme2018/28689 